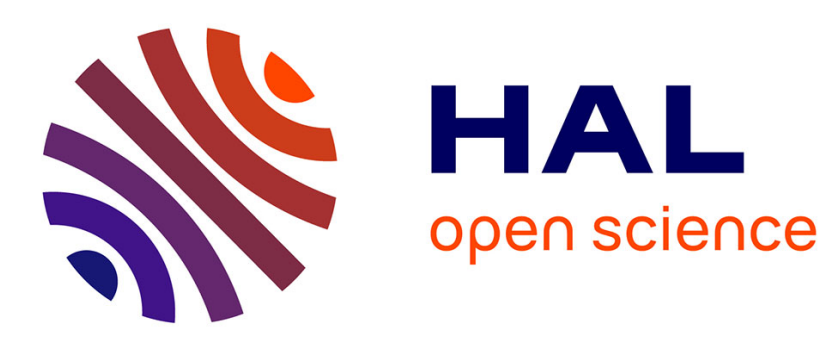

\title{
Digestibilité iléale d'un gluten de blé partiellement hydrolysé chez le veau préruminant
}

\author{
Rs Bush, R Toullec, P Guilloteau, P Barre
}

\section{To cite this version:}

Rs Bush, R Toullec, P Guilloteau, P Barre. Digestibilité iléale d'un gluten de blé partiellement hydrolysé chez le veau préruminant. Annales de zootechnie, 1992, 41 (1), pp.31-32. hal-00888768

\section{HAL Id: hal-00888768 https://hal.science/hal-00888768}

Submitted on 1 Jan 1992

HAL is a multi-disciplinary open access archive for the deposit and dissemination of scientific research documents, whether they are published or not. The documents may come from teaching and research institutions in France or abroad, or from public or private research centers.
L'archive ouverte pluridisciplinaire HAL, est destinée au dépôt et à la diffusion de documents scientifiques de niveau recherche, publiés ou non, émanant des établissements d'enseignement et de recherche français ou étrangers, des laboratoires publics ou privés. 


\title{
Digestibilité iléale d'un gluten de blé partiellement hydrolysé chez le veau préruminant
}

\author{
RS Bush ${ }^{1}, \mathrm{R}$ Toullec ${ }^{1}, \mathrm{P}$ Guilloteau ${ }^{1}, \mathrm{P}$ Barre ${ }^{2}$ \\ 1 INRA, laboratoire du jeune ruminant, 65, rue de Saint-Brieuc, 35042 Rennes Cedex; \\ ${ }^{2}$ UCAAB, BP 19, 02400 Château-Thierry, France
}

\begin{abstract}
Summary - lleal digestibility of a partially hydrolysed wheat gluten in the preruminant calf. The total replacement of casein by a partially hydrolysed wheat gluten in the milk-substitute given to preruminant calves was found to result in a lower apparent and true digestibility at the end of the ileum for nitrogen and most amino acids.
\end{abstract}

Les propriétés fonctionnelles du gluten de blé vital (natif) gênent son emploi dans les aliments d'allaitement qui doivent être distribués sous forme liquide. Une hydrolyse enzymatique partielle permet de résoudre ce problème. Le but de ce travail est d'étudier l'effet du remplacement de la totalité de la caséine par du gluten protéolysé sur la digestibilité iléale.

Matériel et méthodes - Trois veaux préruminants âgés de 2 mois sont munis d'une canule iléale réentrante (Nunes do Prado et al, 1989) et d'un cathéter abomasal. Deux aliments d'allaitement (T et G, 25,5 et $23,7 \%$ de matières azotées (MA) par rapport à la matière sèche (MS)) sont comparés. Dans l'aliment $T$, les MA sont apportées en totalité par de la poudre de lait écrémé. Dans l'aliment $G$, elles sont fournies pour 75,21 et $4 \%$ respectivement par du gluten de blé protéolysé (contenant $75 \%$ de MA/MS), de la poudre de lactosérum et de la L-lysine $\mathrm{HCl}$. Les animaux reçoivent 2 repas par jour, apportant chacun $29 \mathrm{~g}$ de MS/kg (poids vif) ${ }^{2,75}$. Le lait est infusé dans la caillette en 3 à $4 \mathrm{~min}$. Les digesta iléaux sont collectés en totalité pendant $4 j$ avec chacun des aliments. Les profils d'acides aminés (AA) sont comparés par la distance du $\chi^{2}$ (Nunes do Prado et al, 1989).
Résultats et discussion - La digestibilité apparente de l'aliment G est moins élevée que celle de l'aliment $T$ : $0,85 \pm 0,03$ et $0,87 \pm 0,03$ au lieu de $0,92 \pm 0,01$ et $0,93 \pm 0,01$ pour l'azote total et l'azote des AA dosés $(P=0,10$ et 0,08 ). II en est de même pour tous les AA dosés, cystine exceptée (tableau I), pour laquelle la quantité ingérée est 2,2 fois plus forte avec l'aliment $G$. Avec le régime $T$, la composition en AA des digesta est très différente de celle de l'aliment (tableau I), conformément à nos résultats antérieurs montrant que les protéines échappant à la digestion dans l'intestin grêle du veau nourri au lait sont en quasi-totalité d'origine endogène et bactérienne (Nunes do Prado et al, 1989). En revanche, avec le régime $\mathrm{G}$, la composition en $\mathrm{AA}$ des digesta tend à se rapprocher de celle du gluten. Un mélange théorique comprenant $61 \%$ de protéines de gluten et $39 \%$ de protéines de digesta $T$ aurait une composition proche de celle des digesta $G$ : la distance du $\chi^{2}$ ne serait plus que de 39 entre ce mélange et les digesta $G$, au lieu de 255 entre les 2 digesta et de 131 entre les digesta $G$ et le gluten. Dans les digesta, la 
Tableau I. Composition en acides aminés (AA) des aliments et des digesta (\% de la somme des AA dosés). Digestibilité apparente des AA à la fin de l'iléon (moyenne \pm écarts types des moyennes).

\begin{tabular}{|c|c|c|c|c|c|c|c|c|}
\hline \multirow{2}{*}{$A A$} & \multicolumn{3}{|c|}{ Aliment } & \multicolumn{2}{|c|}{ Digesta } & \multicolumn{3}{|c|}{ Digestibilité $\times 100$} \\
\hline & $T$ & $G$ & Gluten & $T$ & $G$ & $S I^{*}$ & $T$ & $G$ \\
\hline Asp & 8,5 & 5,2 & 3,1 & $10,7 \pm 0,5$ & $6,2 \pm 0,3$ & 0,3 & $91 \pm 1$ & $84 \pm 3$ \\
\hline Thr & 4,1 & 3,6 & 2,6 & $7,2 \pm 1,0$ & $4,7 \pm 0,2$ & 1,7 & $88 \pm 1$ & $83 \pm 3$ \\
\hline Ser & 5,2 & 4,7 & 4,6 & $6,0 \pm 0,3$ & $4,0 \pm 0,2^{a}$ & 1,4 & $92 \pm 1$ & $89 \pm 2$ \\
\hline Glu & 20,5 & 31,1 & 36,9 & $17,3 \pm 1,6$ & $31,0 \pm 0,7^{a}$ & 47,4 & $94 \pm 1$ & $87 \pm 2^{a}$ \\
\hline Pro & 9,5 & 10,3 & 12,5 & $5,9 \pm 0,3$ & $10,9 \pm 0,3^{\mathrm{a}}$ & 17,4 & $96 \pm 0$ & $86 \pm 3^{b}$ \\
\hline Gly & 1,9 & 2,9 & 3,1 & $4,8 \pm 0,2$ & $4,8 \pm 0,1$ & 4,8 & $82 \pm 3$ & $78 \pm 4$ \\
\hline Ala & 3,1 & 3,0 & 2,6 & $5,8 \pm 0,6$ & $4,1 \pm 0,1$ & 2,1 & $87 \pm 2$ & $82 \pm 4$ \\
\hline Val & 6,0 & 4,6 & 4,3 & $5,6 \pm 0,0$ & $5,0 \pm 0,2$ & 4,5 & $94 \pm 1$ & $85 \pm 3^{b}$ \\
\hline Cys & 0.8 & 2,0 & 2,1 & $2,9 \pm 0,1$ & $2,8 \pm 0,1$ & 2,8 & $77 \pm 4$ & $81 \pm 4^{a}$ \\
\hline Met & 3,0 & 1,9 & 1,4 & $1,6 \pm 0,2$ & $1,2 \pm 0,1$ & 0,8 & $96 \pm 1$ & $91 \pm 2^{b}$ \\
\hline Ileu & 4,8 & 4,1 & 4,1 & $4,7 \pm 0,3$ & $3,4 \pm 0,1^{b}$ & 1,9 & $93 \pm 1$ & $89 \pm 2$ \\
\hline Leu & 9,4 & 7,5 & 6,9 & $7,9 \pm 0,6$ & $6,5 \pm 0,2$ & 4,8 & $94 \pm 1$ & $88 \pm 2^{b}$ \\
\hline Tyr & 4,8 & 3,0 & 3,4 & $3,6 \pm 0,3$ & $2,4 \pm 0,3^{a}$ & 1,0 & $95 \pm 0$ & $90 \pm 1^{a}$ \\
\hline Phe & 4,7 & 4,6 & 5,3 & $3,9 \pm 0,1$ & $4,0 \pm 0,1$ & 4,0 & $94 \pm 1$ & $89 \pm 2^{a}$ \\
\hline Lys & 7,5 & 6,7 & 1,6 & $6,2 \pm 0,4$ & $4,0 \pm 0,1^{\mathrm{a}}$ & 1,5 & $94 \pm 0$ & $92 \pm 2$ \\
\hline His & 2,7 & 1,8 & 2,1 & $2,3 \pm 0,1$ & $2,0 \pm 0,2$ & 1,9 & $94 \pm 1$ & $85 \pm 5$ \\
\hline Arg & 3,3 & 2,8 & 3,5 & $3,8 \pm 0,2$ & $2,7 \pm 0,2^{\mathrm{a}}$ & 1,4 & $92 \pm 1$ & $87 \pm 4$ \\
\hline$\Sigma A A(g / 16 \mathrm{gN})$ & 102,7 & 96,3 & 101,9 & $86,1 \pm 5,9$ & $85,6 \pm 1,5$ & - & - & - \\
\hline
\end{tabular}

" Supplément d'indigéré dù à l'aliment $G$ par rapport à l'aliment $T_{;}{ }^{a}{ }^{o}$ différence significative entre régimes $(a: P \leq 0,05 ; b$ : $P \leq 0,08)$.

quantité d'azote des $A A$ dosés est en moyenne de 5,7 et $9,8 \mathrm{~g} / 100 \mathrm{~g}$ d'azote total ingéré avec les aliments $T$ et $G$. Le s spplément d'indigéré protidique dû au remplacement de l'aliment $T$ par l'aliment $G$ représente donc $42 \%$ de l'indigéré protidique total obtenu avec cet aliment. II contient beaucoup plus d'acide glutamique et de proline que le gluten (distance du $\chi^{2}=596$ ).

En conclusion, la digestibilité apparente, moins élevée, des protéines du gluten par rapport à celles du lait semble résulter d'une digestibilité réelle incomplète et non d'une augmentation des pertes endogènes.

Remerciements - Ce travail a été effectué avec le soutien du Centre de valorisation des glucides et produits naturels, 6 bis, rue Jean XXIII, 80000 Amiens.

Nunes do Prado I, Toullec R, Guilloteau $P$, Guéguen J (1989) Reprod Nutr Dev 29, 425-439 